\title{
Complete lift of a tensor field of type $(1,2)$ to semi-cotangent bundle
}

\author{
Furkan Yildirim \\ Narman Vocational Training School, Ataturk University, Erzurum, Turkey \\ Received: 16 October 2017, Accepted: 9 November 2017 \\ Published online: 25 December 2017.
}

\begin{abstract}
The main purpose of this paper is to define the complete lift of a projectable tensor field of type $(1,2)$ to semi-cotangent bundle $t^{*} \mathrm{M}$. Using projectable geometric objects on $\mathrm{M}$, we examine lifting problem of projectable tensor field of type $(1,2)$ to the semi-cotangent bundle. We also present the good square in the semi-cotangent bundle $t * \mathrm{M}$.
\end{abstract}

Keywords: Complete lift, pull-back bundle, semi-cotangent bundle, vector field.

\section{Introduction}

Let $M_{n}$ be a differentiable manifold of class $C^{\infty}$ and finite dimension $n$, and let $\left(M_{n}, \pi_{1}, B_{m}\right)$ be a differentiable bundle over $B_{m}$. We use the notation $\left(x^{i}\right)=\left(x^{a}, x^{\alpha}\right)$, where the indices $i, j, \ldots$ run from 1 to $n$, the indices $a, b, \ldots$ from 1 to $n-m$ and the indices $\alpha, \beta, \ldots$ from $n-m+1$ to $n, x^{\alpha}$ are coordinates in $B_{m}, x^{a}$ are fibre coordinates of the bundle

$$
\pi_{1}: M_{n} \rightarrow B_{m}
$$

Let now $\left(T^{*}\left(B_{m}\right), \tilde{\pi}, B_{m}\right)$ be a cotangent bundle [1] over base space $B_{m}$, and let $M_{n}$ be differentiable bundle determined by a natural projection (submersion) $\pi_{1}: M_{n} \rightarrow B_{m}$. The semi-cotangent bundle (pull-back [2], [3], [4], [5], [6]) of the cotangent bundle $\left(T^{*}\left(B_{m}\right), \tilde{\pi}, B_{m}\right)$ is the bundle $\left(t^{*}\left(B_{m}\right), \pi_{2}, M_{n}\right)$ over differentiable bundle $M_{n}$ with a total space

$$
t^{*}\left(B_{m}\right)=\left\{\left(\left(x^{a}, x^{\alpha}\right), x^{\bar{\alpha}}\right) \in M_{n} \times T_{x}^{*}\left(B_{m}\right): \pi_{1}\left(x^{a}, x^{\alpha}\right)=\tilde{\pi}\left(x^{\alpha}, x^{\bar{\alpha}}\right)=\left(x^{\alpha}\right)\right\} \subset M_{n} \times T_{x}^{*}\left(B_{m}\right)
$$

and with the projection map $\pi_{2}: t^{*}\left(B_{m}\right) \rightarrow M_{n}$ defined by $\pi_{2}\left(x^{a}, x^{\alpha}, x^{\bar{\alpha}}\right)=\left(x^{a}, x^{\alpha}\right)$, where $T_{x}^{*}\left(B_{m}\right)\left(x=\pi_{1}(\widetilde{x}), \widetilde{x}=\left(x^{a}, x^{\alpha}\right) \in M_{n}\right)$ is the cotangent space at a point $x$ of $B_{m}$, where $x^{\bar{\alpha}}=p_{\alpha}$ $(\bar{\alpha}, \bar{\beta}, \ldots,=n+1, \ldots, 2 n)$ are fibre coordinates of the cotangent bundle $T^{*}\left(B_{m}\right)$.

Where the pull-back (Pontryagin [7]) bundle $t^{*}\left(B_{m}\right)$ of the differentiable bundle $M_{n}$ also has the natural bundle structure over $B_{m}$, its bundle projection $\pi: t^{*}\left(B_{m}\right) \rightarrow B_{m}$ being defined by $\pi:\left(x^{a}, x^{\alpha}, x^{\bar{\alpha}}\right) \rightarrow\left(x^{\alpha}\right)$, and hence $\pi=\pi_{1} \circ \pi_{2}$. Thus $\left(t^{*}\left(B_{m}\right), \pi_{1} \circ \pi_{2}\right)$ is the composite bundle [[8], p.9] or step-like bundle [9]. Consequently, we notice the semi-cotangent bundle $\left(t^{*}\left(B_{m}\right), \pi_{2}\right)$ is a pull-back bundle of the cotangent bundle over $B_{m}$ by $\pi_{1}$ [6].

If $\left(x^{i^{\prime}}\right)=\left(x^{a^{\prime}}, x^{\alpha^{\prime}}\right)$ is another local adapted coordinates in differentiable bundle $M_{n}$, then we have

$$
\left\{\begin{array}{l}
x^{a^{\prime}}=x^{a^{\prime}}\left(x^{b}, x^{\beta}\right) \\
x^{\alpha^{\prime}}=x^{\alpha^{\prime}}\left(x^{\beta}\right)
\end{array}\right.
$$

\footnotetext{
* Corresponding author e-mail: furkan.yildirim@atauni.edu.tr
} 
The Jacobian of (1) has components

$$
\left(A_{j}^{i^{\prime}}\right)=\left(\frac{\partial x^{i^{\prime}}}{\partial x^{j}}\right)=\left(\begin{array}{cc}
A_{b}^{a^{\prime}} & A_{\beta}^{a^{\prime}} \\
0 & A_{\beta}^{\alpha^{\prime}}
\end{array}\right)
$$

where $A_{b}^{a^{\prime}}=\frac{\partial x^{a^{\prime}}}{\partial x^{b}}, A_{\beta}^{a^{\prime}}=\frac{\partial x^{a^{\prime}}}{\partial x^{\beta}}, A_{\beta}^{\alpha^{\prime}}=\frac{\partial x^{\alpha^{\prime}}}{\partial x^{\beta}}[6]$.

To a transformation (1) of local coordinates of $M_{n}$, there corresponds on $t^{*}\left(B_{m}\right)$ the change of coordinate

$$
\left\{\begin{array}{l}
x^{a^{\prime}}=x^{a^{\prime}}\left(x^{b}, x^{\beta}\right), \\
x^{\alpha^{\prime}}=x^{\alpha^{\prime}}\left(x^{\beta}\right) \\
x^{\bar{\alpha}^{\prime}}=\frac{\partial x^{\beta}}{\partial x^{\alpha^{\prime}}} x^{\beta}
\end{array}\right.
$$

The Jacobian of coordinate system transformation (2) is:

$$
\bar{A}=\left(A_{J}^{I^{\prime}}\right)=\left(\begin{array}{ccc}
A_{b}^{a^{\prime}} & A_{\beta}^{a^{\prime}} & 0 \\
0 & A_{\beta}^{\alpha^{\prime}} & 0 \\
0 & p_{\sigma} A_{\beta}^{\beta^{\prime}} A_{\beta^{\prime} \alpha^{\prime}}^{\sigma} A_{\alpha^{\prime}}^{\beta}
\end{array}\right)
$$

where $I=(a, \alpha, \bar{\alpha}), J=(b, \beta, \bar{\beta}), I, J, \ldots=1, \ldots, 2 n ; A_{\beta^{\prime} \alpha^{\prime}}^{\sigma}=\frac{\partial^{2} x^{\sigma}}{\partial x^{\beta^{\prime}} \partial x^{\alpha^{\prime}}}[6]$.

Now, consider a diagram as

$$
\begin{array}{ccc}
A & \stackrel{\gamma}{\rightarrow} & B \\
\alpha \downarrow & & \downarrow^{\beta} \\
C & \rightarrow & D
\end{array}
$$

A good square of vector bundles is a diagram as above verifying

(i) $\alpha$ and $\beta$ are fibre bundles, but not necessarily vector bundles;

(ii) $\gamma$ and $\pi$ are vector bundles;

(iii) the square is commutative, i.e., $\pi \circ \alpha=\beta \circ \gamma$;

(iv) the local expression

$$
\begin{aligned}
& A \stackrel{\gamma}{\rightarrow} B U^{n} \times R^{r} \times G^{s} \times R^{t} \rightarrow U^{n} \times G^{s}\left(x^{i}, a^{a}, g^{\lambda}, b^{\sigma}\right) \rightarrow\left(x^{i}, g^{\lambda}\right) \\
& { }^{\alpha} \downarrow \downarrow^{\beta} \quad \downarrow \quad \downarrow \quad \downarrow \quad \downarrow \quad \downarrow \\
& C \underset{\pi}{\rightarrow} D \quad U^{n} \times R^{r} \quad \rightarrow \quad U^{n} \quad\left(x^{i}, a^{a}\right) \quad \rightarrow \quad\left(x^{i}\right)
\end{aligned}
$$

where $G$ is a manifold and superindices denote the dimension of the manifolds [11].

By means of above definition, we have

Theorem 1.Let now $\pi: t^{*}\left(B_{m}\right) \rightarrow B_{m}$ be a semi-cotangent bundle and $\pi_{1}: M_{n} \rightarrow B_{m}$ be a fibre bundle. Then, the following is a good square:

$$
\begin{array}{ccccc}
t^{*}\left(B_{m}\right) & \stackrel{\pi_{2}}{\rightarrow} & M_{n} M_{n} \times T_{x}^{*}\left(B_{m}\right) \stackrel{\pi_{2}}{\rightarrow} & M_{n}\left(x^{a}, x^{\alpha}, x^{\bar{\alpha}}\right) \stackrel{\pi_{2}}{\rightarrow}\left(x^{a}, x^{\alpha}\right) \\
i d \downarrow & \downarrow^{\pi_{1}} \quad i d \downarrow & \downarrow^{\pi_{1}} i d \downarrow & \downarrow^{\pi_{1}} \\
t^{*}\left(B_{m}\right) \underset{\pi}{\rightarrow} & B_{m} M_{n} \times T_{x}^{*}\left(B_{m}\right) \underset{\pi}{\rightarrow} B_{m}\left(x^{a}, x^{\alpha}, x^{\bar{\alpha}}\right) \underset{\pi}{\rightarrow}\left(x^{\alpha}\right)
\end{array}
$$

In this study, we continue to study the complete lifts of projectable tensor field of type $(1,2)$ to semi-cotangent (pull-back) bundle $\left(t^{*}\left(B_{m}\right), \pi_{2}\right)$ initiated by F. Yildirim and A. Salimov [6].

We denote by $\mathfrak{I}_{q}^{p}\left(M_{n}\right)$ the set of all tensor fields of class $C^{\infty}$ and of type $(p, q)$ on $M_{n}$, i.e., contravariant degree $p$ and 
covariant degree $q$. We now put $\mathfrak{I}\left(M_{n}\right)=\sum_{p, q=0}^{\infty} \mathfrak{I}_{q}^{p}\left(M_{n}\right)$, which is the set of all tensor fields on $M_{n}$. Smilarly, we denote by $\mathfrak{I}_{q}^{p}\left(B_{m}\right)$ and $\mathfrak{I}\left(B_{m}\right)$ respectively the corresponding sets of tensor fields in the base space $B_{m}$.

Let $\omega$ be a 1 -form with local components $\omega_{\alpha}$ on $B_{m}$, so that $\omega$ is a 1 -form with local expression $\omega=\omega_{\alpha} d x^{\alpha}$. On putting [6]

$$
{ }^{v v} \omega=\left(\begin{array}{l}
0 \\
0 \\
\omega_{\alpha}
\end{array}\right),
$$

we have a vector field ${ }^{v v} \omega$ on $t^{*}\left(B_{m}\right)$. In fact, from (3) we easily see that $\left({ }^{v v} \omega\right)^{\prime}=\bar{A}\left({ }^{v v} \omega\right)$. We call the vector field ${ }^{v v} \omega$ the vertical lift of the 1 -form $\omega$ to $t^{*}\left(B_{m}\right)$.

Let $\widetilde{X} \in \mathfrak{I}_{0}^{1}\left(M_{n}\right)$ be a projectable vector field [10] with projection $X=X^{\alpha}\left(x^{\alpha}\right) \partial_{\alpha}$ i.e. $\widetilde{X}=\widetilde{X}^{a}\left(x^{a}, x^{\alpha}\right) \partial_{a}+X^{\alpha}\left(x^{\alpha}\right) \partial_{\alpha}$. Now, consider $\widetilde{X} \in \mathfrak{I}_{0}^{1}\left(M_{n}\right)$, then ${ }^{c c} \widetilde{X}$ (complete lift) has components on the semi-cotangent bundle $t^{*}\left(B_{m}\right)$ [6]

$$
{ }^{c c} \widetilde{X}=\left({ }^{c} \widetilde{X}^{\alpha}\right)=\left(\begin{array}{l}
\widetilde{X}^{a} \\
X^{\alpha} \\
-p_{\varepsilon}\left(\partial_{\alpha} X^{\varepsilon}\right)
\end{array}\right)
$$

with respect to the coordinates $\left(x^{a}, x^{\alpha}, x^{\bar{\alpha}}\right)$.

\section{$2 \gamma$-operators}

For any $F \in \mathfrak{I}_{1}^{1}\left(B_{m}\right)$, if we take account of (3), we can prove that $(\gamma F)^{\prime}=\bar{A}(\gamma F)$, where $\gamma F$ is a vector field defined by [6]:

$$
\gamma F=\left(\gamma F^{I}\right)=\left(\begin{array}{l}
0 \\
0 \\
p_{\beta} F_{\alpha}^{\beta}
\end{array}\right)
$$

with respect to the coordinates $\left(x^{a}, x^{\alpha}, x^{\bar{\alpha}}\right)$ on $t^{*}\left(B_{m}\right)$.

For any $R \in \mathfrak{I}_{3}^{1}\left(B_{m}\right)$, if we take account of (3), we can prove that $\gamma R_{I^{\prime} J^{\prime}}^{K^{\prime}}=A_{K}^{K^{\prime}} A_{I^{\prime}}^{I} A_{J^{\prime}}^{J} \gamma R_{I J}^{K}$, where $\gamma R$ has components $\bar{R}_{I J}^{K}$ such that

$$
\bar{R}_{\alpha \beta}^{\bar{\gamma}}=P_{\varepsilon} R_{\alpha \beta \gamma}^{\varepsilon}
$$

all the others being zero, with respect to the induced coordinates on $t^{*}\left(B_{m}\right)$. Where $R_{\alpha \beta} \underset{\sigma}{\gamma}$ are local components of $R$ on $B_{m}$ and also $I=(a, \alpha, \bar{\alpha}), J=(b, \beta, \bar{\beta}), K=(c, \gamma, \bar{\gamma})$.

Theorem 2. If $\widetilde{X}$ and $\widetilde{Y}$ be a projectable vector fields on $M_{n}$ with projection $X \in \mathfrak{I}_{0}^{1}\left(B_{m}\right)$ and $Y \in \mathfrak{I}_{0}^{1}\left(B_{m}\right)$. We have

(i) $(\gamma R)\left({ }^{c c} \widetilde{X},{ }^{c c} \widetilde{Y}\right)=\gamma(R(X, Y))$,

(ii) $(\gamma R)\left({ }^{v v} \omega,{ }^{v v} \theta\right)=0$,

(iii) $(\gamma R)\left({ }^{v v} \omega,{ }^{c c} Y\right)=0$,

(iv) $(\gamma R)\left({ }^{v v} \omega, \gamma G\right)=0$,

(v) $(\gamma R)\left({ }^{c c} \widetilde{X}, \gamma G\right)=0$,

(vi) $(\gamma R)(\gamma F, \gamma G)=0$

for any $\omega, \theta \in \mathfrak{I}_{1}^{0}\left(B_{m}\right), F, G \in \mathfrak{I}_{1}^{1}\left(B_{m}\right)$ and $R \in \mathfrak{I}_{3}^{1}\left(B_{m}\right)$. 
Proof. (i) If $R \in \mathfrak{I}_{3}^{1}\left(B_{m}\right), \widetilde{X}$ and $\widetilde{Y}$ be a projectable vector fields on $M_{n}$ with projection $X, Y \in \mathfrak{I}_{0}^{1}\left(B_{m}\right)$ and

$$
\left(\begin{array}{l}
{\left[(\gamma R)\left({ }^{c c} \widetilde{X},{ }^{c c} \widetilde{Y}\right)\right]} \\
{\left[(\gamma R)\left({ }^{c c} \widetilde{X},{ }^{c c} \widetilde{Y}\right)\right]^{\gamma}} \\
{\left[(\gamma R)\left({ }^{c c} \widetilde{X},{ }^{c c} \widetilde{Y}\right)\right]^{\bar{\gamma}}}
\end{array}\right)
$$

are components of $\left[(\gamma R)\left({ }^{c c} \widetilde{X},{ }^{c c} \tilde{Y}\right)\right]^{K}$ with respect to the coordinates $\left(x^{c}, x^{\gamma}, x^{\bar{\gamma}}\right)$ on $t^{*}\left(B_{m}\right)$, then for $K=c$, we have

$$
\left[(\gamma R)\left({ }^{c c} \widetilde{X},{ }^{c c} \widetilde{Y}\right)\right]^{c}=\underbrace{\left(\bar{R}_{\alpha \beta}^{c}\right)}_{0})^{c c} \widetilde{X}^{\alpha c c} \widetilde{Y}^{\beta}=0
$$

because of (5) and (7). For $K=\gamma$, we have

$$
\left[(\gamma R)\left({ }^{c c} \widetilde{X},{ }^{c c} \widetilde{Y}\right)\right]^{\gamma}=\underbrace{\left(\bar{R}_{\alpha}^{\gamma}\right)^{c c}}_{0} \widetilde{X}^{\alpha c c} \widetilde{Y}^{\beta}=0
$$

because of (5) and (7). For $K=\bar{\gamma}$, we have

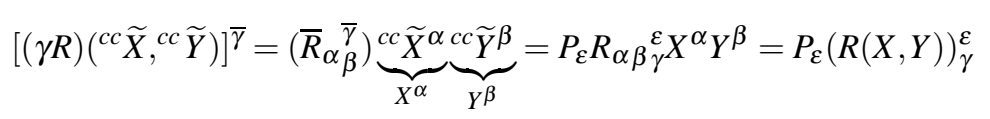

because of (5) and (7). It is well known that $\gamma(R(X, Y))$ have components

$$
\gamma(R(X, Y))=\left(\begin{array}{l}
0 \\
0 \\
P_{\varepsilon}(R(X, Y))_{\gamma}^{\varepsilon}
\end{array}\right)
$$

with respect to the coordinates $\left(x^{c}, x^{\gamma}, x^{\bar{\gamma}}\right)$ on $t^{*}\left(B_{m}\right)$. Thus, we have $(\gamma R)\left({ }^{c c} \widetilde{X},{ }^{c c} \widetilde{Y}\right)=\gamma(R(X, Y))$. Similarly, we can easily compute another equations of Theorem 2 .

\section{Complete lift of a tensor field of type $(1,2)$ to semi-cotangent bundle}

Let $\widetilde{S} \in \mathfrak{I}_{2}^{1}\left(M_{n}\right)$ be a projectable tensor field of type $(1,2)$ with projection $S=S_{i j}^{k}\left(x^{a}, x^{\alpha}\right) \partial_{k} \otimes d x^{i} \otimes d x^{j}$, i.e. $\widetilde{S}$ has componets such that

$$
{ }^{c c} \widetilde{S}_{\alpha \beta}^{c}=S_{\alpha \beta}^{c}
$$

with respect to the coordinates on $M_{n}$. Where $i=(a, \alpha), j=(b, \beta), k=(c, \gamma)$.

If we take account of (3), we can prove that ${ }^{c c} \widetilde{S}_{I^{\prime}} K_{J^{\prime}}^{\prime}=A_{K}^{K^{\prime}} A_{I^{\prime}}^{I} A_{J^{\prime}}^{J} c c \widetilde{S}_{I_{J}}^{K}$, where ${ }^{c c} \widetilde{S}$ has components ${ }^{c c} \widetilde{S}_{I_{J}}^{K}$ such that

$$
\left\{\begin{array}{l}
{ }^{c c} \widetilde{S}_{\alpha \beta}^{c}=S_{\alpha \beta}^{c} \\
{ }^{c c} \widetilde{S}_{\alpha}^{\gamma}=S_{\alpha \beta}^{\gamma} \\
{ }^{c c} \widetilde{S}_{\alpha}^{\frac{\beta}{\gamma}}=-p_{\varepsilon}\left(\partial_{\alpha} S_{\beta \gamma}^{\varepsilon}+\partial_{\beta} S_{\gamma \alpha}^{\varepsilon}+\partial_{\gamma} S_{\alpha \beta}^{\varepsilon}\right) \\
{ }^{c c} \widetilde{S}_{\alpha} \frac{\beta}{\gamma}=S_{\alpha \gamma}^{\beta} \\
{ }^{c c} \widetilde{S}_{\bar{\alpha}}^{\frac{\beta}{\gamma}}=S_{\gamma \beta}^{\alpha}
\end{array}\right.
$$

all the others being zero, with respect to the induced coordinates on $t^{*}\left(B_{m}\right)$. Where $S_{I J}^{K}$ are local components of $S$ on $M_{n}$ and also $I=(a, \alpha, \bar{\alpha}), J=(b, \beta, \bar{\beta}), K=(c, \gamma, \bar{\gamma})$. 
Proof. For convenience sake we only consider ${ }^{c c} \widetilde{S}_{\alpha_{\prime}^{\prime} \beta^{\prime}} \bar{\gamma}^{\prime}$ In fact,

$$
{ }^{c c} \widetilde{S}_{\overline{\alpha^{\prime}} \beta^{\prime}}^{\bar{\gamma}^{\prime}}=A_{\bar{\gamma}}^{\bar{\gamma}^{\prime}} A_{\bar{\alpha}^{\prime}}^{\bar{\alpha}} A_{\beta^{\prime}}^{\beta c c} \widetilde{S}_{\bar{\alpha}}^{\bar{\gamma}}=A_{\gamma^{\prime}}^{\gamma} A_{\alpha}^{\alpha^{\prime}} A_{\beta^{\prime}}^{\beta} S_{\gamma \beta}^{\alpha}=S_{\gamma^{\prime} \beta^{\prime}}^{\alpha^{\prime}}
$$

Thus, we have ${ }^{c c} \widetilde{S}_{\bar{\alpha}}^{\bar{\gamma}}=S_{\gamma \beta}^{\alpha}$. Similarly, from (3) and (8), we can easily find all other components of ${ }^{c c} \widetilde{S}_{I J}^{K}$ equal to zero, where $I=(a, \alpha, \bar{\alpha}), J=(b, \beta, \bar{\beta}), K=(c, \gamma, \bar{\gamma})$.

Theorem 3. Let $\widetilde{S} \in \mathfrak{I}_{2}^{1}\left(M_{n}\right)$ be a projectable tensor field of type (1,2). If $\widetilde{X}, \widetilde{Y} \in \mathfrak{I}_{0}^{1}\left(M_{n}\right), \omega, \theta \in \mathfrak{I}_{1}^{0}\left(B_{m}\right), F, G \in \mathfrak{I}_{1}^{1}\left(B_{m}\right)$ then

(i) ${ }^{c c} \widetilde{S}\left({ }^{v v} \omega,{ }^{v v} \theta\right)=0$,

(ii) ${ }^{c c} \widetilde{S}\left({ }^{v v} \omega, \gamma G\right)=0$,

(iii) ${ }^{c c} \widetilde{S}\left({ }^{v v} \omega,{ }^{c c} \widetilde{Y}\right)=-{ }^{v v}\left(\omega \circ S_{Y}\right)$,

(iv) ${ }^{c c} \widetilde{S}(\gamma F, \gamma G)=0$

(v) ${ }^{c c} \widetilde{S}\left(\gamma F,{ }^{c c} \widetilde{Y}\right)=-\gamma\left(F \circ S_{Y}\right)$,

(vi) ${ }^{c c} \widetilde{S}\left({ }^{c c} \widetilde{X},{ }^{c c} \widetilde{Y}\right)={ }^{c c}(S(X, Y))-\gamma\left(\left(L_{X} S\right)_{Y}-\left(L_{Y} S\right)_{X}+S_{[X, Y]}\right)$,

where $L_{X} S$ denotes the Lie derivative of $S$ with respect to $X$.

Proof. (i) If $\omega, \theta \in \mathfrak{I}_{1}^{0}\left(B_{m}\right)$ and $\widetilde{S}$ is projectable tensor field of type $(1,2)$ on $M_{n}$ with projection $S \in \mathfrak{I}_{2}^{1}\left(B_{m}\right)$ and

$$
\left(\begin{array}{c}
\left({ }^{c c} \widetilde{\boldsymbol{S}}\left({ }^{v v} \omega,{ }^{v v} \theta\right)\right)^{c} \\
\left({ }^{c c} \widetilde{\boldsymbol{S}}\left({ }^{v v} \omega,{ }^{v v} \theta\right)\right)^{\gamma} \\
\left({ }^{c c} \widetilde{\boldsymbol{S}}\left({ }^{v v} \omega,{ }^{v v} \theta\right)\right)^{\gamma}
\end{array}\right)
$$

are components of $\left({ }^{c c} \widetilde{S}\left({ }^{v v} \omega,{ }^{v v} \theta\right)\right)^{K}$ with respect to the coordinates $\left(x^{c}, x^{\gamma}, x^{\bar{\gamma}}\right)$ on $t^{*}\left(B_{m}\right)$, then we have

$$
\left({ }^{c c} \widetilde{S}\left({ }^{v v} \omega,{ }^{v v} \theta\right)\right)^{K}={ }^{c c} \widetilde{S}_{I J}^{K v v} \omega^{I v v} \theta^{J}={ }^{c c} \widetilde{S}_{\bar{\alpha}} \frac{K v}{\beta} \omega^{\bar{\alpha} v v} \theta^{\bar{\beta}}={ }^{c c} \widetilde{S}_{\bar{\alpha}} \frac{K}{\beta} \omega_{\alpha} \theta_{\beta}
$$

Firstly, if $K=c$, we have

$$
\left({ }^{c c} \widetilde{S}\left({ }^{v v} \omega,{ }^{v v} \theta\right)\right)^{c}=\underbrace{c c}_{0} \widetilde{S}_{\bar{\alpha} \frac{c}{\beta}} \omega_{\alpha} \theta_{\beta}=0
$$

by virtue of (4) and (8). Secondly, if $K=\gamma$, we have

$$
\left({ }^{c c} \widetilde{S}\left({ }^{v v} \omega,{ }^{v v} \theta\right)\right)^{\gamma}=\underbrace{{ }^{c c} \widetilde{S_{\bar{\alpha}}} \frac{\gamma}{\beta}}_{0} \omega_{\alpha} \theta_{\beta}=0
$$

by virtue of (4) and (8). Thirdly, if $J=\bar{\beta}$, then we have

$$
\left({ }^{c c} \widetilde{S}\left({ }^{v v} \omega,{ }^{v v} \theta\right)\right)^{\bar{\gamma}}=\underbrace{{ }^{c c} \widetilde{S_{\alpha}} \frac{\bar{\gamma}}{\beta}}_{0} \omega_{\alpha} \theta_{\beta}=0
$$

by virtue of (4) and (8). Thus (i) of Theorem 3 is proved. 
(ii) If $G \in \mathfrak{I}_{1}^{1}\left(B_{m}\right)$ and $\widetilde{S}$ is projectable tensor field of type $(1,2)$ on $M_{n}$ with projection $S \in \mathfrak{I}_{2}^{1}\left(B_{m}\right)$ and

$$
\left(\begin{array}{l}
\left({ }^{c c} \widetilde{S}\left({ }^{v v} \omega, \gamma G\right)\right)^{c} \\
\left({ }^{c c} \widetilde{S}\left({ }^{v v} \omega, \gamma G\right)\right)^{\gamma} \\
\left({ }^{c c} \widetilde{S}\left({ }^{v v} \omega, \gamma G\right)\right)^{\gamma}
\end{array}\right)
$$

are components of $\left.\left({ }^{c c} \widetilde{S}{ }^{v v} \omega, \gamma G\right)\right)^{K}$ with respect to the coordinates $\left(x^{c}, x^{\gamma}, x^{\bar{\gamma}}\right)$ on $t^{*}\left(B_{m}\right)$, then we have

$$
\left({ }^{c c} \widetilde{S}\left({ }^{v v} \omega, \gamma G\right)\right)^{K}={ }^{c c} \widetilde{S}_{I J}^{K v v} \omega^{I} \gamma G^{J}={ }^{c c} \widetilde{S}_{\bar{\alpha}} \bar{K}^{v v} \omega^{\bar{\alpha}} \gamma G^{\bar{\beta}}={ }^{c c} \widetilde{S}_{\bar{\alpha}} \frac{K}{\bar{\beta}} \omega_{\alpha} p_{\varepsilon} G_{\beta}^{\varepsilon} .
$$

Firstly, if $K=c$, we have

$$
\left({ }^{c c} \widetilde{S}\left({ }^{v v} \omega, \gamma G\right)\right){ }^{c}=\underbrace{{ }^{c c} \widetilde{S_{\alpha}} \frac{c}{\beta}}_{0} \omega_{\alpha} p_{\varepsilon} G_{\beta}^{\varepsilon}=0
$$

by virtue of (4), (6) and (8). Secondly, if $K=\gamma$, we have

$$
\left({ }^{c c} \widetilde{S}\left({ }^{v v} \omega, \gamma G\right)\right)^{\gamma}=\underbrace{c c}_{0} \widetilde{S}_{\bar{\alpha}} \frac{\gamma}{\beta} \omega_{\alpha} p_{\varepsilon} G_{\beta}^{\varepsilon}=0
$$

by virtue of (4), (6) and (8). Thirdly, if $J=\bar{\beta}$, then we have

$$
\left({ }^{c c} \widetilde{S}\left({ }^{v v} \omega, \gamma G\right)\right)^{\bar{\gamma}}=\underbrace{{ }^{c c} \widetilde{S_{\bar{\alpha}}} \frac{\bar{\gamma}}{\bar{\beta}}}_{0} \omega_{\alpha} p_{\varepsilon} G_{\beta}^{\varepsilon}=0
$$

by virtue of (4), (6) and (8). Thus (ii) of Theorem 3 is proved.

(iii) If $\widetilde{Y} \in \mathfrak{I}_{0}^{1}\left(M_{n}\right)$ and $\widetilde{S}$ is projectable tensor field of type $(1,2)$ on $M_{n}$ with projection $S \in \mathfrak{I}_{2}^{1}\left(B_{m}\right)$ and

$$
\left(\begin{array}{c}
\left({ }^{c c} \widetilde{\boldsymbol{S}}\left({ }^{v v} \omega,{ }^{c c} \widetilde{Y}\right)\right)^{c} \\
\left({ }^{c c} \widetilde{\boldsymbol{S}}\left({ }^{v v} \omega,{ }^{c c} \widetilde{Y}\right)\right)^{\gamma} \\
\left({ }^{c c} \widetilde{\boldsymbol{S}}\left({ }^{v v} \omega,{ }^{c c} \widetilde{Y}\right)\right)^{\gamma}
\end{array}\right)
$$

are components of $\left({ }^{c c} \widetilde{S}\left({ }^{v v} \omega,{ }^{c c} \widetilde{Y}\right)\right)^{K}$ with respect to the coordinates $\left(x^{c}, x^{\gamma}, x^{\bar{\gamma}}\right)$ on $t^{*}\left(B_{m}\right)$, then we have

$$
\left({ }^{c c} \widetilde{S}\left({ }^{v v} \omega{ }^{c c} \widetilde{Y}\right)\right)^{K}={ }^{c c} \widetilde{S}_{I J}^{K}\left({ }^{v v} \omega\right)^{I}\left({ }^{c c} \widetilde{Y}\right)^{J}={ }^{c c} \widetilde{S}_{\bar{\alpha} b}^{K}\left({ }^{v v} \omega\right)^{\bar{\alpha}}\left({ }^{c c} \widetilde{Y}\right)^{b}+{ }^{c c} \widetilde{S}_{\bar{\alpha} \beta}^{K}\left({ }^{v v} \omega\right)^{\bar{\alpha}}\left({ }^{c c} \widetilde{Y}\right)^{\beta}+{ }^{c c} \widetilde{S}_{\bar{\alpha}} \frac{K}{\bar{\beta}}\left({ }^{v v} \omega\right)^{\bar{\alpha}}\left({ }^{c c} \widetilde{Y}\right)^{\bar{\beta}}
$$

Firstly, if $K=c$, we have

$$
\left({ }^{c c} \widetilde{S}\left({ }^{v v} \omega,{ }^{c c} \widetilde{Y}\right)\right)^{c}=\underbrace{c c}_{0} \widetilde{S}_{\bar{\alpha} b}^{c}\left({ }^{c v} \omega\right)^{\bar{\alpha}}\left({ }^{c c} \widetilde{Y}\right)^{b}+\underbrace{{ }^{c c} \widetilde{S}_{\bar{\alpha}}{ }^{c}}_{0}\left({ }^{v v} \omega\right)^{\bar{\alpha}}\left({ }^{c c} \widetilde{Y}\right)^{\beta}+\underbrace{{ }^{c c} \widetilde{S}_{\bar{\alpha}} \frac{c}{\bar{\beta}}}_{0}{ }^{\left({ }^{v v}\right.} \omega)^{\bar{\alpha}}\left({ }^{c c} \widetilde{Y}\right)^{\bar{\beta}}=0
$$


by virtue of (4), (5) and (8). Secondly, if $K=\gamma$, we have

$$
\left({ }^{c c} \widetilde{S}\left({ }^{v v} \omega,{ }^{c c} \widetilde{Y}\right)\right)^{\gamma}=\underbrace{c c \widetilde{S}_{\bar{\alpha}}^{\gamma}}_{0}\left({ }^{v v} \omega\right)^{\bar{\alpha}}\left({ }^{c c} \widetilde{Y}\right)^{b}+\underbrace{{ }^{c c} \widetilde{S}_{\bar{\alpha}}^{\gamma}}_{0}{ }^{\gamma}{ }^{v v} \omega)^{\bar{\alpha}}\left({ }^{c c} \widetilde{Y}\right)^{\beta}+\underbrace{{ }^{c c} \widetilde{S}_{\bar{\alpha}} \frac{\gamma}{\beta}}_{0}\left({ }^{v v} \omega\right)^{\bar{\alpha}}\left({ }^{c c} \widetilde{Y}\right)^{\bar{\beta}}=0
$$

by virtue of (4), (5) and (8). Thirdly, if $K=\bar{\gamma}$, then we have

$$
\begin{aligned}
\left({ }^{c c} \widetilde{S}\left({ }^{v v} \omega,{ }^{c c} \widetilde{Y}\right)\right)^{\bar{\gamma}} & =\underbrace{c c}_{0} \widetilde{S}_{\bar{\alpha}}^{\bar{\gamma}}\left({ }^{v v} \omega\right)^{\bar{\alpha}}\left({ }^{c c} \widetilde{Y}\right)^{b}+\underbrace{{ }^{c c} \widetilde{S}_{\bar{\alpha}}^{\bar{\gamma}}}_{S_{\gamma \beta}^{\alpha}=-S_{\beta \gamma}^{\alpha}}\left({ }^{v v} \omega\right)^{\bar{\alpha}}\left({ }^{c c} \widetilde{Y}\right)^{\beta}+\underbrace{{ }^{c c} \widetilde{S}_{\bar{\alpha}} \bar{\beta}}_{0}\left({ }^{v v} \omega\right)^{\bar{\alpha}}\left({ }^{c c} \widetilde{Y}\right)^{\bar{\beta}} \\
& =-S_{\beta \gamma}^{\alpha} \omega_{\alpha} Y^{\beta}=-S_{\beta \gamma}^{\alpha} \omega_{\alpha} Y^{\beta}=-\left(\omega \circ S_{Y}\right)_{\gamma}
\end{aligned}
$$

by virtue of (4), (5) and (8). On the other hand, we know that ${ }^{v v}\left(\omega \circ S_{Y}\right)$ have components

$$
{ }^{v v}\left(\omega \circ S_{Y}\right)=\left(\begin{array}{l}
0 \\
0 \\
\left(\omega \circ S_{Y}\right)_{\gamma}
\end{array}\right)
$$

with respect to the coordinates $\left(x^{c}, x^{\gamma}, x^{\bar{\gamma}}\right)$ on $t^{*}\left(B_{m}\right)$. Thus, we have ${ }^{c c} \widetilde{S}\left({ }^{v v} \omega,{ }^{c c} \widetilde{Y}\right)=-{ }^{v v}\left(\omega \circ S_{Y}\right)$.

(iv) If $F, G \in \mathfrak{I}_{1}^{1}\left(B_{m}\right)$ and $\widetilde{S}$ is projectable tensor field of type $(1,2)$ on $M_{n}$ with projection $S \in \mathfrak{I}_{2}^{1}\left(B_{m}\right)$ and

$$
\left(\begin{array}{c}
\left({ }^{c} \widetilde{S}(\gamma F, \gamma G)\right)^{c} \\
\left({ }^{c} \widetilde{S}(\gamma F, \gamma G)\right)^{\gamma} \\
\left({ }^{c} \widetilde{S}(\gamma F, \gamma G)\right)^{\gamma}
\end{array}\right)
$$

are components of $\left({ }^{c c} \widetilde{S}(\gamma F, \gamma G)\right)^{K}$ with respect to the coordinates $\left(x^{c}, x^{\gamma}, x^{\bar{\gamma}}\right)$ on $t^{*}\left(B_{m}\right)$, then we have

$$
\left({ }^{c c} \widetilde{S}(\gamma F, \gamma G)\right)^{K}=\quad \quad{ }^{c c} \widetilde{S}_{I J}^{K} \gamma F^{I} \gamma G^{J}={ }^{c c} \widetilde{S}_{\bar{\alpha}} \frac{K}{\beta}(\gamma F)^{\bar{\alpha}}(\gamma G)^{\bar{\beta}}={ }^{c c} \widetilde{S}_{\bar{\alpha}} \frac{K}{\beta}\left(p_{\varepsilon} F_{\alpha}^{\varepsilon}\right)\left(p_{\varepsilon} G_{\beta}^{\varepsilon}\right) .
$$

Firstly, if $K=c$, we have

$$
\left({ }^{c c} \widetilde{S}(\gamma F, \gamma G)\right)^{c}=\underbrace{c c}_{0} \widetilde{S}_{\bar{\alpha}} \frac{c}{\beta}\left(p_{\varepsilon} F_{\alpha}^{\varepsilon}\right)\left(p_{\varepsilon} G_{\beta}^{\varepsilon}\right)=0
$$

by virtue of (6) and (8). Secondly, if $K=\gamma$, we have

$$
\left({ }^{c c} \widetilde{S}(\gamma F, \gamma G)\right)^{\gamma}=\underbrace{{ }^{c c} \widetilde{S}_{\bar{\alpha}} \frac{\gamma}{\beta}}_{0}\left(p_{\varepsilon} F_{\alpha}^{\varepsilon}\right)\left(p_{\varepsilon} G_{\beta}^{\varepsilon}\right)=0
$$

by virtue of (6) and (8). Thirdly, if $J=\bar{\beta}$, then we have

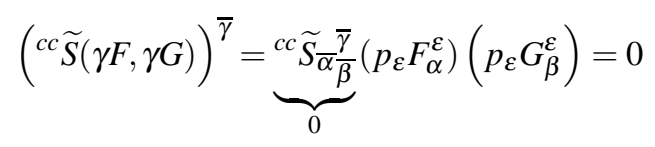


by virtue of (6) and (8). Thus (iv) of Theorem 3 is proved.

(v) If $\widetilde{Y} \in \mathfrak{I}_{0}^{1}\left(M_{n}\right)$ and $\widetilde{S}$ is projectable tensor field of type $(1,2)$ on $M_{n}$ with projection $S \in \mathfrak{I}_{2}^{1}\left(B_{m}\right)$ and

$$
\left(\begin{array}{c}
\left({ }^{c c} \widetilde{S}\left(\gamma F,{ }^{c c} \widetilde{Y}\right)\right)^{c} \\
\left({ }^{c c} \widetilde{S}\left(\gamma F,{ }^{c c} \widetilde{Y}\right)\right)^{\gamma} \\
\left({ }^{c c} \widetilde{S}\left(\gamma F,{ }^{c c} \widetilde{Y}\right)\right)^{\gamma}
\end{array}\right)
$$

are components of $\left({ }^{c c} \widetilde{S}\left(\gamma F,{ }^{c c} \widetilde{Y}\right)\right)^{K}$ with respect to the coordinates $\left(x^{c}, x^{\gamma}, x^{\bar{\gamma}}\right)$ on $t^{*}\left(B_{m}\right)$, then we have

$$
\left({ }^{c c} \widetilde{S}\left(\gamma F,{ }^{c c} \widetilde{Y}\right)\right)^{K}={ }^{c c} \widetilde{S}_{I J}^{K}(\gamma F)^{I}\left({ }^{c c} \widetilde{Y}\right)^{J}={ }^{c c} \widetilde{S}_{\bar{\alpha} b}{ }^{K}(\gamma F)^{\bar{\alpha}}\left({ }^{c c} \widetilde{Y}\right)^{b}+{ }^{c c} \widetilde{S}_{\bar{\alpha}}{ }_{\beta}^{K}(\gamma F)^{\bar{\alpha}}\left({ }^{c c} \widetilde{Y}\right)^{\beta}+{ }^{c c} \widetilde{S}_{\bar{\alpha}} \frac{K}{\beta}(\gamma F)^{\bar{\alpha}}\left({ }^{c c} \widetilde{Y}\right)^{\bar{\beta}} .
$$

Firstly, if $K=c$, we have

$$
\left({ }^{c c} \widetilde{S}\left(\gamma F,{ }^{c c} \widetilde{Y}\right)\right)^{c}=\underbrace{c c}_{0} \widetilde{S}_{\bar{\alpha} b}^{c}(\gamma F)^{\bar{\alpha}}\left({ }^{c c} \widetilde{Y}\right)^{b}+\underbrace{{ }^{c c} \widetilde{S}_{\bar{\alpha}}^{c}}_{0}(\gamma F)^{\bar{\alpha}}\left({ }^{c c} \widetilde{Y}\right)^{\beta}+\underbrace{{ }^{c c} \widetilde{S}_{\bar{\alpha}} \frac{c}{\beta}}_{0}(\gamma F)^{\bar{\alpha}}\left({ }^{c c} \widetilde{Y}\right)^{\bar{\beta}}=0
$$

by virtue of (5), (6) and (8). Secondly, if $K=\gamma$, we have

$$
\left({ }^{c c} \widetilde{S}\left(\gamma F,{ }^{c c} \widetilde{Y}\right)\right)^{\gamma}=\underbrace{c c}_{0} \widetilde{S}_{\bar{\alpha}}^{\gamma}(\gamma F)^{\bar{\alpha}}\left({ }^{c c} \widetilde{Y}\right)^{b}+\underbrace{{ }^{c c} \widetilde{S}_{\bar{\alpha}}^{\gamma}}_{0}(\gamma F)^{\bar{\alpha}}\left({ }^{c c} \widetilde{Y}\right)^{\beta}+\underbrace{{ }^{c c} \widetilde{S}_{\bar{\alpha}}^{\gamma} \frac{\gamma}{\beta}}_{0}(\gamma F)^{\bar{\alpha}}\left({ }^{c c} \widetilde{Y}\right)^{\bar{\beta}}=0
$$

by virtue of (5), (6) and (8). Thirdly, if $K=\bar{\gamma}$, then we have

$$
\begin{aligned}
\left({ }^{c c} \widetilde{S}\left(\gamma F,{ }^{c c} \widetilde{Y}\right)\right)^{\bar{\gamma}} & =\underbrace{{ }^{c c} \widetilde{S}_{\bar{\alpha}}^{\bar{\gamma}}}_{0}(\gamma F)^{\bar{\alpha}}\left({ }^{c c} \widetilde{Y}\right)^{b}+\underbrace{}_{S_{\gamma_{\beta}^{\alpha}=-S_{\beta \gamma}^{\alpha}}{ }^{c c} \widetilde{S}_{\bar{\alpha}}^{\bar{\gamma}}}(\gamma F)^{\bar{\alpha}}\left({ }^{c c} \widetilde{Y}\right)^{\beta}+\underbrace{{ }^{c c} \widetilde{S}_{\bar{\alpha}} \frac{\bar{\gamma}}{\beta}}_{0}(\gamma F)^{\bar{\alpha}}\left({ }^{c c} \widetilde{Y}\right)^{\bar{\beta}} \\
& =-S_{\beta \gamma}^{\alpha} p_{\varepsilon} F_{\alpha}^{\varepsilon} Y^{\beta}=-p_{\varepsilon}\left(S_{\beta \gamma}^{\alpha} F_{\alpha}^{\varepsilon} Y^{\beta}\right)=-p_{\varepsilon}\left(F \circ S_{Y}\right)_{\gamma}^{\varepsilon}
\end{aligned}
$$

by virtue of (5), (6) and (8). On the other hand, we know that $\gamma\left(F \circ S_{Y}\right)$ have components

$$
\gamma\left(F \circ S_{Y}\right)=\left(\begin{array}{l}
0 \\
0 \\
p_{\varepsilon}\left(F \circ S_{Y}\right)_{\gamma}^{\varepsilon}
\end{array}\right)
$$

with respect to the coordinates $\left(x^{c}, x^{\gamma}, x^{\bar{\gamma}}\right)$ on $t^{*}\left(B_{m}\right)$. Thus, we have ${ }^{c c} \widetilde{S}\left(\gamma F,{ }^{c c} \widetilde{Y}\right)=-\gamma\left(F \circ S_{Y}\right)$.

(vi) If $\widetilde{X}, \widetilde{Y} \in \mathfrak{I}_{0}^{1}\left(M_{n}\right)$ and $\widetilde{S}$ is projectable tensor field of type $(1,2)$ on $M_{n}$ with projection $S \in \mathfrak{I}_{2}^{1}\left(B_{m}\right)$ and

$$
\left(\begin{array}{c}
\left({ }^{c c} \widetilde{S}\left({ }^{c c} \widetilde{X},{ }^{c c} \tilde{Y}\right)\right)^{c} \\
\left({ }^{c c} \widetilde{S}\left({ }^{c c} \widetilde{X},{ }^{c c} \widetilde{Y}\right)\right)^{\gamma} \\
\left({ }^{c c} \widetilde{S}\left({ }^{c c} \widetilde{X},{ }^{c c} \tilde{Y}\right)\right)^{\gamma}
\end{array}\right)
$$


are components of $\left({ }^{c c} \widetilde{S}\left({ }^{c c} \widetilde{X},{ }^{c c} \widetilde{Y}\right)\right)^{K}$ with respect to the coordinates $\left(x^{c}, x^{\gamma}, x^{\bar{\gamma}}\right)$ on $t^{*}\left(B_{m}\right)$, then we have

$$
\left.\left({ }^{c c} \widetilde{S}\left({ }^{c c} \widetilde{X},{ }^{c c} \widetilde{Y}\right)\right)^{K}={ }^{c c} \widetilde{S}_{I J}^{K}\left({ }^{c c} \widetilde{X}\right)^{I}\left({ }^{c c} \widetilde{Y}\right)^{J}={ }^{c c} \widetilde{S}_{\alpha \beta}{ }^{k c} \widetilde{X}\right)^{\alpha}\left({ }^{c c} \widetilde{Y}\right)^{\beta}+{ }^{c c} \widetilde{S}_{\alpha} \frac{K}{\beta}\left({ }^{c c} \widetilde{X}\right)^{\alpha}\left({ }^{c c} \widetilde{Y}\right)^{\bar{\beta}}+{ }^{c c} \widetilde{S}_{\bar{\alpha} \beta}{ }^{K}\left({ }^{c c} \widetilde{X}\right)^{\bar{\alpha}}\left({ }^{c c} \widetilde{Y}\right)^{\beta} .
$$

Firstly, if $K=c$, we have

$$
\begin{aligned}
\left({ }^{c c} \widetilde{S}\left({ }^{c c} \widetilde{X},{ }^{c c} \widetilde{Y}\right)\right)^{c} & =\underbrace{\left({ }^{c c} \widetilde{X}\right)^{\alpha}}_{S_{\alpha_{\beta}^{c}}^{c c} \widetilde{S}_{\alpha \beta}^{c}} \underbrace{\left({ }^{c c} \widetilde{Y}\right)^{\beta}}_{X^{\alpha}}+\underbrace{{ }^{c c} \widetilde{S}_{\alpha} \frac{c}{\beta}}_{0}\left({ }^{c c} \widetilde{X}\right)^{\alpha}\left({ }^{c c} \widetilde{Y}\right)^{\bar{\beta}}+\underbrace{{ }^{c c} \widetilde{S}_{\bar{\alpha} \beta}^{c}}_{0}\left({ }^{c c} \widetilde{X}\right)^{\bar{\alpha}}\left({ }^{c c} \widetilde{Y}\right)^{\beta} \\
& =S_{\alpha \beta}^{c} X^{\alpha} Y^{\beta}=(S(X, Y))^{c}
\end{aligned}
$$

by virtue of (5) and (8). Secondly, if $K=\gamma$, we have

$$
\begin{aligned}
\left({ }^{c c} \widetilde{S}\left({ }^{c c} \widetilde{X},{ }^{c c} \widetilde{Y}\right)\right)^{\gamma} & =\underbrace{\gamma}_{S_{\alpha_{\beta}^{\gamma}}{ }^{c} \widetilde{S}_{\alpha}^{\gamma}} \underbrace{\left({ }^{c c} \widetilde{X}\right)^{\alpha}}_{X^{\alpha}} \underbrace{\left({ }^{c c} \widetilde{Y}\right)^{\beta}}_{Y^{\beta}}+\underbrace{{ }^{c c} \widetilde{S}_{\alpha} \frac{\gamma}{\beta}}_{0}\left({ }^{c c} \widetilde{X}\right)^{\alpha}\left({ }^{c c} \widetilde{Y}\right)^{\bar{\beta}}+\underbrace{c c}_{0} \widetilde{S}_{\bar{\alpha}}^{\gamma}\left({ }^{c c} \widetilde{X}\right)^{\bar{\alpha}}\left({ }^{c c} \widetilde{Y}\right)^{\beta} \\
& =S_{\alpha_{\beta}}^{\gamma} X^{\alpha} Y^{\beta}=(S(X, Y))^{\gamma}
\end{aligned}
$$

by virtue of (5) and (8). Thirdly, if $K=\bar{\gamma}$, then we have

$$
\begin{aligned}
\left({ }^{c c} \widetilde{S}\left({ }^{c c} \widetilde{X},{ }^{c c} \widetilde{Y}\right)\right)^{\bar{\gamma}} & ={ }^{c c} \widetilde{S}_{\alpha \beta}^{\bar{\gamma}}\left({ }^{c c} \widetilde{X}\right)^{\alpha}\left({ }^{c c} \widetilde{Y}\right)^{\beta}+{ }^{c c} \widetilde{S}_{\alpha} \bar{\gamma} \bar{\beta}\left({ }^{c c} \widetilde{X}\right)^{\alpha}\left({ }^{c c} \widetilde{Y}\right)^{\bar{\beta}}+{ }^{c c} \widetilde{S}_{\bar{\alpha}}^{\bar{\gamma}}\left({ }^{c c} \widetilde{X}\right)^{\bar{\alpha}}\left({ }^{c c} \widetilde{Y}\right)^{\beta} \\
& =-p_{\varepsilon}\left(\partial_{\alpha} S_{\beta}{ }_{\gamma}^{\varepsilon}+\partial_{\beta} S_{\gamma \alpha}^{\varepsilon}+\partial_{\gamma} S_{\alpha}{ }_{\alpha}^{\varepsilon}\right) X^{\alpha} Y^{\beta}-p_{\varepsilon} S_{\alpha \gamma}^{\beta} X^{\alpha} \partial_{\beta} Y^{\varepsilon}-p_{\varepsilon} S_{\gamma \beta}^{\alpha} \partial_{\alpha} X^{\varepsilon} Y^{\beta} \\
& =-p_{\varepsilon} \partial_{\alpha} S_{\beta}^{\varepsilon} X^{\alpha} Y^{\beta}-p_{\varepsilon} \partial_{\beta} S_{\gamma \alpha}^{\varepsilon} X^{\alpha} Y^{\beta}-p_{\varepsilon} \partial_{\gamma} S_{\alpha \beta}^{\varepsilon} X^{\alpha} Y^{\beta}-p_{\varepsilon} S_{\alpha \gamma}^{\beta} X^{\alpha} \partial_{\beta} Y^{\varepsilon}-p_{\varepsilon} S_{\gamma \beta}^{\alpha} \partial_{\alpha} X^{\varepsilon} Y^{\beta} \\
& =-\underbrace{p_{\alpha} \partial_{\beta} S_{\varepsilon \gamma}^{\alpha} X^{\beta} Y^{\varepsilon}}_{A 1}-\underbrace{p_{\alpha} \partial_{\varepsilon} S_{\gamma \beta}^{\alpha} X^{\beta} Y^{\varepsilon}}_{A 2}-\underbrace{p_{\alpha} \partial_{\gamma} S_{\beta \varepsilon}^{\alpha} X^{\beta} Y^{\varepsilon}}_{A 3}-\underbrace{p_{\varepsilon} S_{\alpha \gamma}^{\beta} X^{\alpha} \partial_{\beta} Y^{\varepsilon}}_{A 4}+\underbrace{p_{\varepsilon} S_{\beta \gamma}^{\alpha} \partial_{\alpha} X^{\varepsilon} Y^{\beta}}_{A 5}
\end{aligned}
$$

by virtue of (5) and (8). We know that ${ }^{c c}(S(X, Y))^{\bar{\gamma}}, p_{\alpha}\left(\left(L_{X} S\right)_{Y}\right)_{\gamma}^{\alpha},-p_{\alpha}\left(\left(L_{Y} S\right)_{X}\right)_{\gamma}^{\alpha}$ and $p_{\alpha}\left(S_{[X, Y]}\right)_{\gamma}^{\alpha}$ have respectively, components on $t^{*}\left(B_{m}\right)$

$$
\begin{aligned}
&{ }^{c c}(S(X, Y))^{\bar{\gamma}}=-p_{\alpha} \partial_{\gamma}\left(S_{\beta \varepsilon}^{\alpha} X^{\beta} Y^{\varepsilon}\right)=-p_{\alpha}\left(\partial_{\gamma} S_{\beta \varepsilon}^{\alpha}\right) X^{\beta} Y^{\varepsilon}-p_{\alpha}\left(\partial_{\gamma} X^{\beta}\right) S_{\beta \varepsilon}^{\alpha} Y^{\varepsilon}-p_{\alpha}\left(\partial_{\gamma} Y^{\varepsilon}\right) S_{\beta \varepsilon}^{\alpha} X^{\beta} \\
& c c(S(X, Y))^{\bar{\gamma}}=-p_{\alpha}\left(\partial_{\gamma} S_{\beta \varepsilon}^{\alpha}\right) X^{\beta} Y^{\varepsilon}+p_{\alpha}\left(\partial_{\gamma} X^{\beta}\right) S_{\varepsilon \beta}^{\alpha} Y^{\varepsilon}-p_{\alpha}\left(\partial_{\gamma} Y^{\varepsilon}\right) S_{\beta \varepsilon}^{\alpha} X^{\beta} \\
& c c(S(X, Y))^{\bar{\gamma}}=\underbrace{-p_{\alpha}\left(\partial_{\gamma} S_{\beta \varepsilon}^{\alpha}\right) X^{\beta} Y^{\varepsilon}}_{A 3}+\underbrace{p_{\alpha}\left(\partial_{\gamma} X^{\beta}\right) S_{\varepsilon \beta}^{\alpha} Y^{\varepsilon}}_{A 6}-\underbrace{p_{\alpha}\left(\partial_{\gamma} Y^{\varepsilon}\right) S_{\beta \varepsilon}^{\alpha} X^{\beta}}_{A 7} \\
& p_{\alpha}\left(\left(L_{X} S\right)_{Y}\right)_{\gamma}^{\alpha}=\underbrace{p_{\alpha} X^{\beta} \partial_{\beta} S_{\varepsilon \gamma}^{\alpha} Y^{\varepsilon}}_{A 1}+\underbrace{p_{\alpha} \partial_{\varepsilon} X^{\beta} S_{\beta \gamma}^{\alpha} Y^{\varepsilon}}_{A 8}+\underbrace{p_{\alpha} \partial_{\gamma} X^{\beta} S_{\varepsilon \beta}^{\alpha} Y^{\varepsilon}}_{A 6}-\underbrace{p_{\alpha} \partial_{\beta} X^{\alpha} S_{\varepsilon \gamma}^{\beta} Y^{\varepsilon}}_{A 5}, \\
&-p_{\alpha}\left(\left(L_{Y} S\right)_{X}\right)_{\gamma}^{\alpha}=-\underbrace{p_{\alpha} Y^{\beta} \partial_{\beta} S_{\varepsilon \gamma}^{\alpha} X^{\varepsilon}}_{A 2}-\underbrace{p_{\alpha} \partial_{\varepsilon} Y^{\beta} S_{\beta \gamma}^{\alpha} X^{\varepsilon}}_{A 9}-\underbrace{p_{\alpha} \partial_{\gamma} Y^{\beta} S_{\varepsilon \beta}^{\alpha} X^{\varepsilon}}_{A 7}+\underbrace{p_{\alpha} \partial_{\beta} Y^{\alpha} S_{\varepsilon \gamma}^{\beta} X^{\varepsilon}}_{A 7}, \\
&\left.p_{\alpha}\left(S_{[X, Y]}\right)_{\gamma}^{\alpha}=p_{\alpha}^{S_{\beta \gamma}^{\alpha}\left(X^{\varepsilon} \partial_{\varepsilon} Y^{\beta}\right.}-Y^{\varepsilon} \partial_{\varepsilon} X^{\beta}\right)=\underbrace{p_{\alpha} S_{\beta \gamma}^{\alpha} X^{\varepsilon} \partial_{\varepsilon} Y^{\beta}}_{A 9}-\underbrace{p_{\alpha} S_{\beta \gamma}^{\alpha} Y^{\varepsilon} \partial_{\varepsilon} X^{\beta}}_{A 8}
\end{aligned}
$$


with respect to the coordinates $\left(x^{c}, x^{\gamma}, x^{\bar{\gamma}}\right)$. Where the same equations are denoted by $A 1, A 2, \ldots, A 9$. On the other hand, we know that ${ }^{c c}(S(X, Y))$ and $\gamma\left(\left(L_{X} S\right)_{Y}-\left(L_{Y} S\right)_{X}+S_{[X, Y]}\right)$ have respectively, components

$$
\begin{aligned}
{ }^{c c}(S(X, Y))= & \left(\begin{array}{l}
(S(X, Y))^{c} \\
(S(X, Y))^{\gamma} \\
-p_{\varepsilon} \partial_{\gamma}(S(X, Y))^{\varepsilon}
\end{array}\right) \\
\gamma\left(\left(L_{X} S\right)_{Y}-\left(L_{Y} S\right)_{X}+S_{[X, Y]}\right)= & \left(\begin{array}{l}
0 \\
0 \\
p_{\alpha}\left(\left(L_{X} S\right)_{Y}-\left(L_{Y} S\right)_{X}+S_{[X, Y]}\right)_{\gamma}^{\alpha}
\end{array}\right)
\end{aligned}
$$

with respect to the coordinates $\left(x^{c}, x^{\gamma}, x^{\bar{\gamma}}\right)$ on $t^{*}\left(B_{m}\right)$. Thus, we have

$$
{ }^{c c} \widetilde{S}\left({ }^{c c} \widetilde{X},{ }^{c c} \widetilde{Y}\right)={ }^{c c}(S(X, Y))-\gamma\left(\left(L_{X} S\right)_{Y}-\left(L_{Y} S\right)_{X}+S_{[X, Y]}\right)
$$

by the necessary simplifications made in equalities.

\section{Competing interests}

The authors declare that they have no competing interests.

\section{Authors' contributions}

All authors have contributed to all parts of the article. All authors read and approved the final manuscript.

\section{References}

[1] K. Yano and S. Ishihara, Tangent and Cotangent Bundles. Marcel Dekker, Inc., New York, 1973.

[2] D. Husemoller, Fibre Bundles. Springer, New York, 1994.

[3] H.B. Lawson and M.L. Michelsohn, Spin Geometry. Princeton University Press., Princeton, 1989.

[4] N. Steenrod, The Topology of Fibre Bundles. Princeton University Press., Princeton, 1951.

[5] F. Y1ldırım, On a special class of semi-cotangent bundle, Proceedings of the Institute of Mathematics and Mechanics, (ANAS) 41 (2015), no. 1, 25-38.

[6] F. Yildırım and A. Salimov, Semi-cotangent bundle and problems of lifts, Turk J. Math, (2014), 38, 325-339.

[7] L.S. Pontryagin, Characteristic cycles on differentiable manifolds. Rec. Math. (Mat. Sbornik) N.S., 21 (63):2, (1947), $233-284$.

[8] W.A. Poor, Differential Geometric Structures, New York, McGraw-Hill (1981).

[9] N.M. Ostianu, Step-fibred spaces, Tr. Geom. Sem. 5, Moscow. (VINITI), 259-309 (1974).

[10] V. V. Vishnevskii, Integrable affinor structures and their plural interpretations. Geometry, 7.J. Math. Sci. (New York) 108 (2002), no. 2, 151-187.

[11] F. Etayo, The geometry of good squares of vector bundles, Riv. Mat. Univ. Parma 17 (1991) 131-147. 